\title{
Interaction of Dyes with Cationic Surfactants in Solutions: Determination of Critical Micelle Concentration
}

\author{
S. Shapovalov* and V. Ponomariov \\ Institute of Chemistry, V. N. Karazin Kharkiv National University, Kharkiv, 61022, Ukraine \\ *serghey.a.shapovalov@karazin.ua
}

\begin{abstract}
Keywords: dyes, sulforhodamine B, ethyl eosin, surfactants, critical micelle concentration, spectroscopy, cetylpyridinium bromide, solution.
\end{abstract}

\begin{abstract}
Interactions of sulforhodamine B or ethyl eosin (as the anionic dye) with cetylpyridinium bromide (as a cationic surfactant) have been investigated by visible spectroscopy in aqueous solution. The object of research is the association between an anion of dye and of cationic surfactant in aqueous solutions and its features. Computer simulation of these interactions was carried out with the use of AM1 and PM3 semi-empirical methods. A new spectrophotometric method was developed for the determination of critical micelle concentration of cationic surfactants.
\end{abstract}

\section{Introduction}

Recently, much attention is paid to the study of the phenomena of the self- or dissimilar association (i.e. heteroassociation) of organic dyes in aqueous and mixed media [1-3]. The association significantly affects on the spectral and luminescent properties of different systems. For example, the ionic association with the participation of dyes finds a fruitful application for the quantitative determination of metal ions, surfactants and organic additives [4-6]. Features of the association are also investigated in the biomedical industry, in pharmaceutical chemistry in order to determine the components in drugs or modification of DNA and polypeptides [7,8].

The association of the "dye - surfactant" type has a special interest due to certain features (the presence of spectral changes at sufficiently low concentrations of reagents at the level of $10^{-6} \mathrm{M}$ and even less). In general, association models and thermodynamics (association constants, enthalpy of formation and Gibbs energy) and other parameters (surface tension, the surface area per surfactant molecule at the water/air interface and others) were determined for different classes of dyes [9-19]. There are studies to find effective ways to extract some dyes from various media using surfactants [20-23].

Its own unique properties attract the attention of xanthene dyes, among them rhodamines and oxyxanthenes $[1,3,24]$. They are characterized by high intensity of light absorption of charged protolytic forms and the ability to intense luminescence in solutions. Our investigation is focused on (i) interaction of sulforhodamine B or ethyl eosin anions with cationic surfactant in water, (ii) assessment of energy interaction "dye-surfactant", (iii) consideration of the possibility of developing a simple method for quantifying the critical micelle concentration for cationic surfactant. These results should be helpful for understanding the "dye-surfactant" interaction at the molecular level and be useful for practical purposes.

\section{Experimental}

\subsection{Materials and reagents}

Sulforhodamine B, SR (CAS Number: 3520-42-1, Colour Index Number: 45100), ethyl eosin, EE (CAS Number: 6359-05-3, Colour Index Number: 45386), cetylpyridinium bromide, CPB (CAS Number: 140-72-7) were purchased from Sigma-Aldrich Chemical Company, USA, and used without further purification. Chemical structures of the investigated particles are presented on Fig. 1. 
<smiles>CCN(CC)c1ccc2c(-c3ccc(S(=O)(=O)[O-])cc3S(=O)(=O)[O-])c3ccc(=[N+](CC)CC)cc-3oc2c1</smiles>

(a)<smiles>CCOC(=O)c1ccccc1-c1c2cc(Br)c(=O)c(Br)c-2oc2c(Br)c(OC)c(Br)cc12</smiles>

(b)<smiles>C[n+]1ccccc1</smiles>

(c)

Figure 1. Chemical structure of SR (a) and EE (b) anions and CPB (c).

\subsection{Solutions}

SR and EE solutions were prepared by dissolving the appropriate weight in $100 \mathrm{~cm}^{3}$ water. CPB solution was prepared by dissolving the appropriate weight in $250 \mathrm{~cm}^{3}$ distilled water. Photometric flasks contained molar concentrations of dyes from $1 \cdot 10^{-6}$ to $5 \cdot 10^{-5} \mathrm{M}$ and no more because to avoid the process of possible dimerization. Solutions of dyes were stored in dark glass bottles to be protected from light. Doubly distilled water was used for the preparation of aqueous solutions.

\subsection{Apparatus}

Absorption spectral measurements were carried out with a UV-visible modernized spectrophotometer SF-46. The spectrophotometric measurements were carried out using $1 \mathrm{~cm}$ glass cell, with wavelength range of $410-600 \mathrm{~nm}$. The experiments were carried out at room temperature.

\section{Results and Discussion}

\subsection{Properties of the dyes and surfactant}

Considered dyes form singly charged ions $\mathrm{R}^{-}$in aqueous solutions. These anions exist on a fairly wide range of $\mathrm{pH}$ values in accordance with the thermodynamic $\mathrm{p} K_{\mathrm{a}}$ values (3.22 for SR [24] and 1.80 for EE [1]). Sufficiently large values of the molar absorption coefficients $(\varepsilon)$ of the anions $\left(108000 \mathrm{~L} \cdot \mathrm{mol}^{-1} \cdot \mathrm{cm}^{-1}\right.$ for SR $(565 \mathrm{~nm})$ and $98000 \mathrm{~L} \cdot \mathrm{mol}^{-1} \cdot \mathrm{cm}^{-1}$ for EE $\left.(520 \mathrm{~nm})\right)$ make it possible to investigate the interactions with surfactant at fairly low molar particle concentrations $\left(\sim 10^{-6} \mathrm{M}\right)$. $\mathrm{CPB}$ exists in the form of a single charge cation $\mathrm{CP}^{+}$in such concentrations (the value of $\mathrm{CCM}$ equals to $\left.(1.0 \div 9.0) \cdot 10^{-4} \mathrm{M}[25-27]\right)$.

Interpretation of the spectral changes within the equilibrium approach implies the implementation of the main law of light absorption by the protolytic forms of the dyes. It was experimentally determined that the linear dependence of the optical density, $A$, on the concentration of dye in solution can be applied satisfactorily over quite wide ranges of molar concentration, $C$, of the anions:

$$
\begin{aligned}
& A_{565}=-0.0487_{(0.034)}+1.01110^{5}{ }_{(350)} \cdot C(\text { for SR }) \\
& A_{520}=-0.0079_{(0.004)}+9.73010^{4}{ }_{(330)} \cdot C(\text { for EE })
\end{aligned}
$$

The values of free regression term are statistically zero (standard deviation of each parameter is given in brackets), and it could be considered that $A_{\lambda}=\mathrm{k} \cdot C$. It is worth noting the practical coincidence of correlation coefficient with the unity. This gives reason to suggest that anions do not dimerize in the studied concentration intervals $\left(1 \cdot 10^{-6} \mathrm{M}-5 \cdot 10^{-5} \mathrm{M}\right)$. Thus, the spectral changes that are instrumentally recorded when mixing the dye and surfactant are due to the interaction of the anion with the surfactant cation. 


\subsection{Absorption spectra and energetics of "dye-surfactant" interaction}

Supplementary experiments have been revealed that additions of buffer solutions (phosphate, borate, acetate solutions) did not have a noticeable influence on the "dye-surfactant" interaction. Spectral changes in the systems of "dye-surfactant" are due only to the interaction of anion dye with a cation of surfactant (see Fig. 2, Fig. 3).

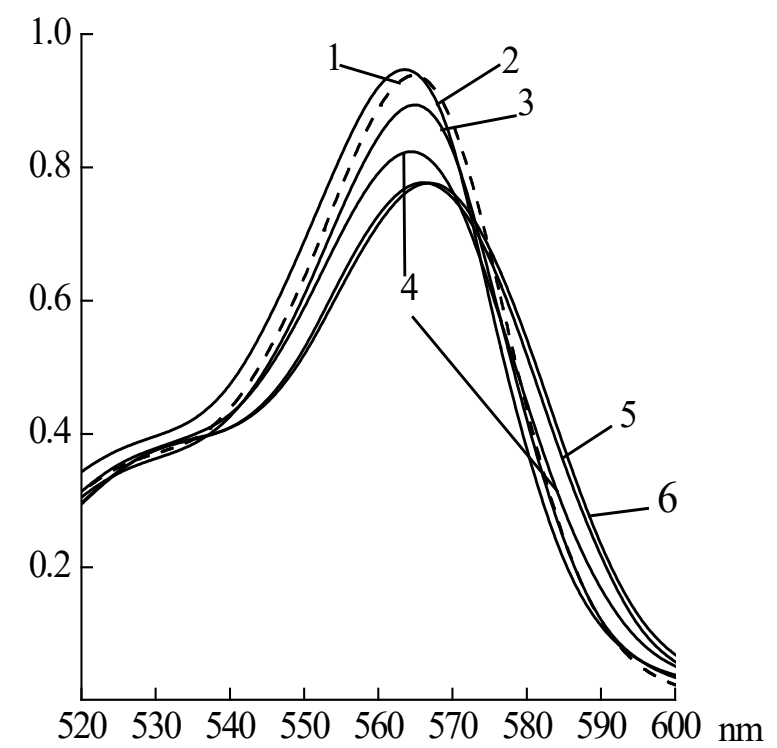

Figure 2. Absorption spectra of SR solution $\left(8.5 \cdot 10^{-6} \mathrm{M}\right)$ vs. the CPB concentration, M: $0(1)$, $1.0 \cdot 10^{-6}(2), 1 \cdot 10^{-4}(3), 4.02 \cdot 10^{-4}(4), 9.05 \cdot 10^{-4}(5), 9.45 \cdot 10^{-4}(6)$. The optical path length is $1.0 \mathrm{~cm}$.

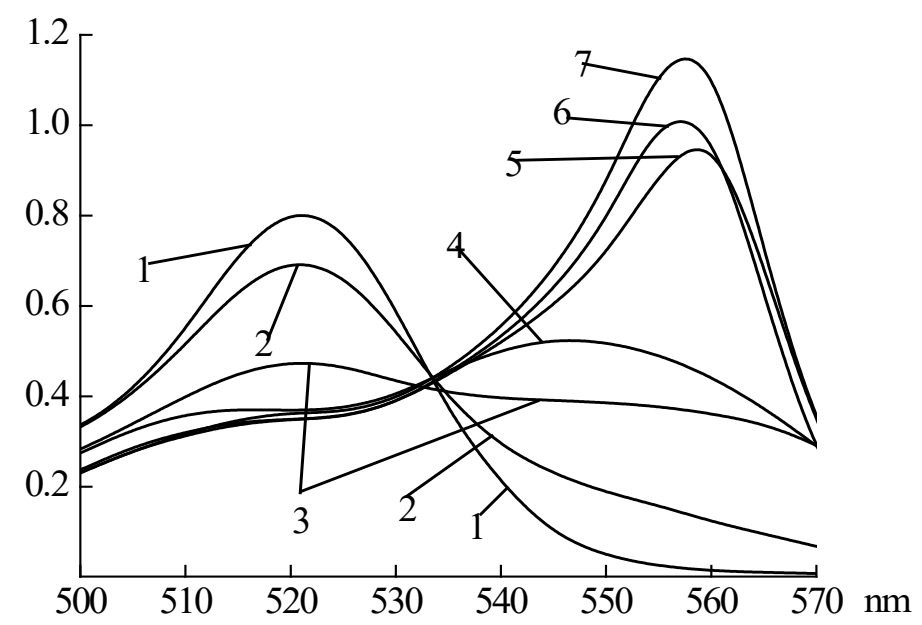

Figure 3. Absorption spectra of EE solution $\left(8.6 \cdot 10^{-6} \mathrm{M}\right)$ vs. the CPB concentration, $\mathrm{M}$ : 0 (1), $1.05 \cdot 10^{-5}(2), 3.14 \cdot 10^{-5}(3), 5.23 \cdot 10^{-5}-5.23 \cdot 10^{-4}(4), 1.05 \cdot 10^{-3}(5), 1.20 \cdot 10^{-3}(6), 1.36 \cdot 10^{-3}(7)$. The optical path length is $1.0 \mathrm{~cm}$.

Similar spectral changes are typical for other concentrations $\left(2.1 \cdot 10^{-6}, 4.3 \cdot 10^{-6}, 1.1 \cdot 10^{-5} \mathrm{M}\right)$ of each dye. This fact indicates the interaction of the surfactant cation with the dye anion. It is found that the absorption spectra do not change over time even at different molar concentrations of both dyes. The decrease in the intensity of absorption (spectra 1-3 on Fig. 3) indicates the presence of processes of the association of " $\mathrm{CP}^{+}+\mathrm{R}^{-}$" type. However, further increase in surfactant concentration until the micelles leads to an increase in light absorption and evidence of solubilization dye (spectra 5-7 on Fig. 3). This experimental fact is in agreement with the modern ideas of the existence of the dye in micelles systems of ionic surfactants $[1,3,14]$. 
Molecular modelling of the systems " $\mathrm{CP}^{+}+\mathrm{R}^{-}$" and related calculations were performed using the package "HyperChem 8.0" [28] for a variety of initial options for the location of counterions relative to each other ("single point" procedure). General principles of quantum-chemical simulations of the structures have been described previously [29, 30]. Geometric optimization of ions was carried out by molecular mechanics MM+ (vacuum; see Fig. 4 as an example) and AMBER (water, "Periodic Box" function) methods.

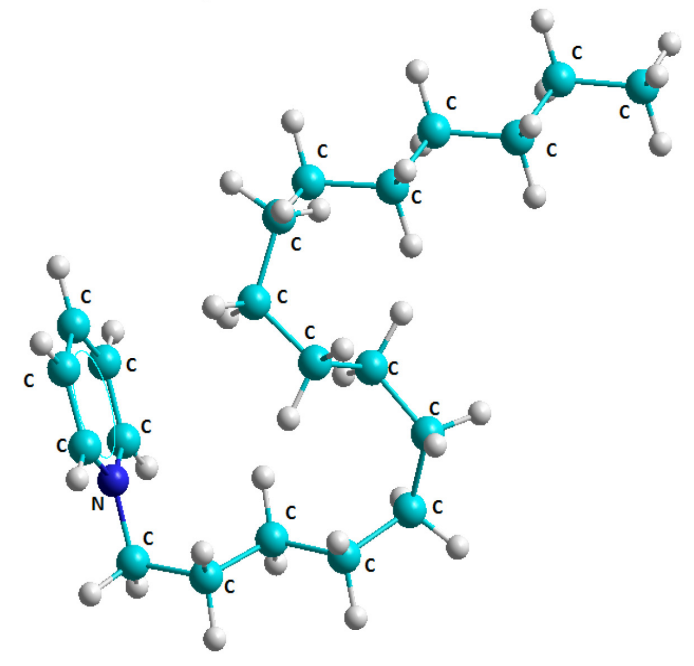

Figure 4. The optimized structure of $\mathrm{CP}^{+}$.

Standard enthalpy $\left(\Delta \mathrm{H}^{0}\right)$ of formation of ions and associate " $\mathrm{CP}^{+}+\mathrm{R}^{-"}$ was determined by semi-empirical methods AM1 and PM3. The parameters of these methods were selected so that they allow us to reproduce the experimental values of $\Delta \mathrm{H}^{0}$ of organic compounds in the best way. The use of two methods of simulation is related not only to the tendency to assess the higher reliability in absolute values of $\Delta \mathrm{H}^{0}$, but also to minimize the systematic error in the discovery of the change in for associates of similar composition. As an example, Table 1 shows the characteristics of the " $\mathrm{CP}^{+}+\mathrm{SR}^{-"}$ interaction depending on the distance between the Nitrogen atom within a pair and is denoted in the first column, an Oxygen atom (or phenolic fragment of the structure of SR') in the composition of the dye. The variants with the highest cation-anion interaction energy gain are shown in bold.

Table 1. The characteristics of the " $\mathrm{CP}^{+}+\mathrm{SR}^{-"}$ interaction.

\begin{tabular}{|c|c|c|c|c|c|c|c|c|}
\hline \multirow{2}{*}{$\begin{array}{c}\text { Distance } \\
\text { N(CP } \\
\text { fragment, } \AA\end{array}$} & \multicolumn{5}{|c|}{$\Delta(\Delta \mathrm{H})=\Sigma \Delta \mathrm{H}^{0}$ ions $\left(\mathrm{CP}^{+}+\mathrm{SR}^{-}\right)-\Delta \mathrm{H}^{0}$ associate, $\mathrm{kcal} / \mathrm{mol}$} \\
\cline { 2 - 10 } & \multicolumn{3}{|c|}{ vacuum } & \multicolumn{4}{c|}{ water } \\
\cline { 2 - 10 } & $\Delta \mathrm{H}^{0}$ & $\Delta\left(\Delta \mathrm{H}^{0}\right)$ & $\Delta \mathrm{H}^{0}$ & $\Delta\left(\Delta \mathrm{H}^{0}\right)$ & $\Delta \mathrm{H}^{0}$ & $\Delta\left(\Delta \mathrm{H}^{0}\right)$ & $\Delta \mathrm{H}^{0}$ & $\Delta\left(\Delta \mathrm{H}^{0}\right)$ \\
\hline $\mathbf{5 . 2}-\mathbf{P h}$ & $\mathbf{- 3 2}$ & $\mathbf{8 8}$ & $\mathbf{- 5 4}$ & $\mathbf{8 7}$ & - & - & - & - \\
\hline $5.1-\mathrm{Ph}$ & -24 & 80 & -47 & 80 & 2894 & -171 & 2465 & -55 \\
\hline $5.4-\mathrm{Ph}$ & 23 & 34 & -5 & 38 & 3409 & 344 & 2861 & 341 \\
\hline $4.4-\mathrm{Ph}$ & -12 & 68 & -42 & 75 & 2987 & -78 & 2363 & -157 \\
\hline $\mathbf{4 . 8}-\mathbf{O}$ & 39 & 17 & 11 & 22 & $\mathbf{3 3 7 8}$ & $\mathbf{3 1 3}$ & $\mathbf{2 8 7 4}$ & $\mathbf{3 5 4}$ \\
\hline $4.2-\mathrm{O}$ & 33 & 23 & 7 & 26 & 2932 & -133 & 2453 & -67 \\
\hline
\end{tabular}

The average error in calculation of $\Delta \mathrm{H}^{0}$ is about $6 \mathrm{kcal} / \mathrm{mol}$ [31]. The "single point" procedure states that the optimized structures of the associate " $\mathrm{CP}^{+}+\mathrm{SR}^{-"}$ for vacuum and water box have different $\Delta \mathrm{H}^{0}$ values since the organic cation and anion are differently positioned relative to each 
other. But the results of simulations show that the association of the dye with surfactant is energetically favorable, since the value of the standard enthalpy of formation of associates " $\mathrm{CP}^{+}+$ $\mathrm{SR}^{-"}$ and "CP$+\mathrm{EE}^{+}$" is much less than the total value of $\Delta \mathrm{H}^{0}$ for each of the ions $\left(\mathrm{SR}^{-}\right.$

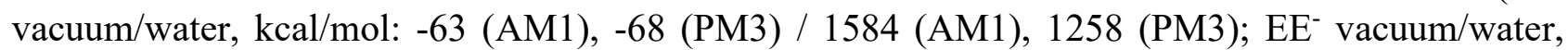
kcal/mol: -125 (AM1), -134 (PM3) / 1038 (AM1), 876 (PM3); $\mathrm{CP}^{+}$vacuum/water, kcal/mol: 119 (AM1), 131 (PM3) / 1481 (AM1), 1262 (PM3)). Both methods (AM1, PM3) give almost identical values of $\Delta\left(\Delta \mathrm{H}^{0}\right)$ in the case of vacuum $(88$ and $87 \mathrm{kcal} / \mathrm{mol}$, respectively). Note, that in the case of a water environment, the $\Delta\left(\Delta \mathrm{H}^{0}\right)$ have even more significant but fairly close values (313 and $354 \mathrm{kcal} / \mathrm{mol})$.

\subsection{Determination of critical micelle concentration}

Known methods are currently used to quantify the cationic surfactant CMC in water, based on a sharp change in the physical and chemical properties of surfactant solutions, such as optical density, with an increase in the concentration of surfactants from the molecular solution to the micellar. The $\mathrm{CMC}$ value is determined by the location of the fracture point in the graphic dependence of the optical density on the surfactant concentration in water. This relationship has the form of two straight sections intersecting. The disadvantage of the methods is the insufficient reliability of the QMS determination, which is due to the blurring of the fracture point location on the graphic dependences due to the insufficient linearity of the plots. This leads to the undesirable need to further involve other instrumental methods in order to more reliably determine the CMC of cationic surfactants, for example, the method of surface tension or electrical conductivity. The elimination of the noted drawback in the known methods is associated with the use of dyes that can significantly reflect the change in optical density depending on the concentration of cationic surfactants in water $[12,26]$.

We have determined that SR is able to interact with cationic surfactant (CPB) in water in such a way that there is a change in the intensity of light absorption, behind which it becomes possible to determine the CMC. The method for determining the CMC involves the use of SR, the subsequent measurement of the optical density $A$ at a certain wavelength and the construction of a graphical dependence of $A$ at a wavelength of light absorption of $565 \mathrm{~nm}$ from the concentration of cationic surfactants (Fig. 5).

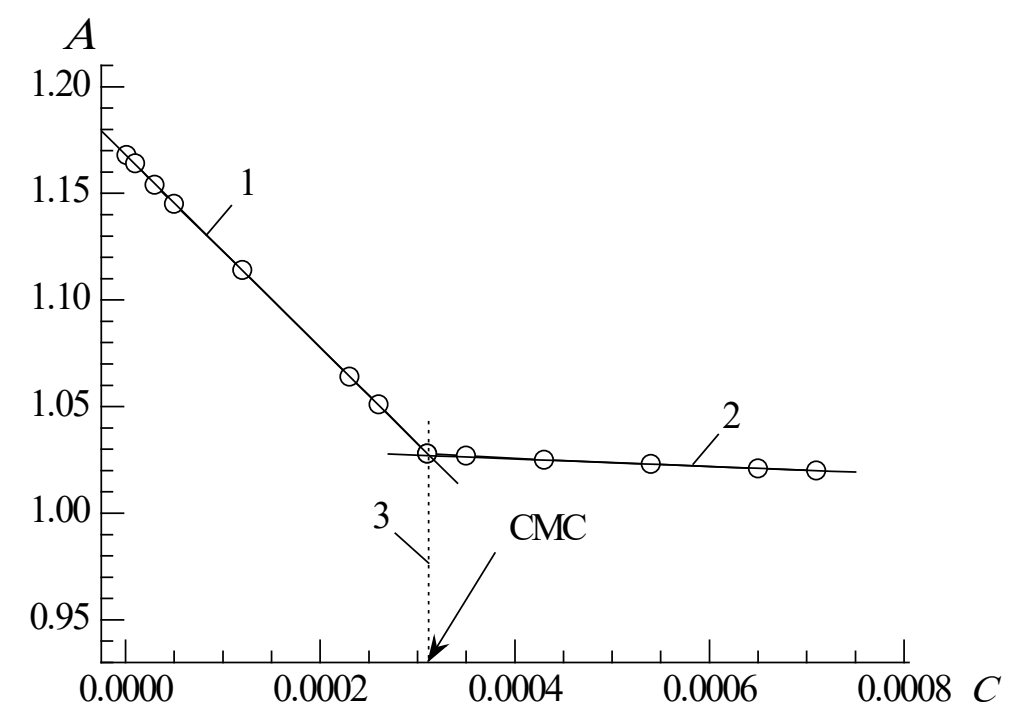

Figure 5. The dependence of the optical density on the content of CPB in water. The wavelength of light absorption is $565 \mathrm{~nm}$.

Type of dependence of optical density on the concentration of CPB can be explained as follows. The highest optical density is characteristic of the dye in the absence of CPB. When adding CPB to a constant amount of SR optical density decreases in direct proportion to the molar surfactant concentration (area 1) due to the formation of a compound between the dye and CPB. 
With the subsequent increase in the concentration of CPB solution is converted from molecular to micellar. The optical density ceases to decrease (area 2) since the amount of dye that interacted with the CPB does not change. Under these conditions, SR is completely in the micelles of CPB, regardless of the further increase in the concentration of surfactants in water. Perpendicular 3 determines the minimum surfactant concentration at which the solution becomes micellar, that is, cuts off the CMC value of cationic pairs on the abscissa axis. The value $3.2 \cdot 10^{-4}$ for CMC of CPB is found from the graph, and it coincides well with the known values [25]. But due to the greater certainty of the fracture point on the dependence (Fig. 5), the new method is able to determine the $\mathrm{CMC}$ of cationic surfactants with better reliability in comparison with some spectrophotometric methods $[12,25,26]$.

\section{Conclusions}

The changes in absorption spectra caused by the interaction between the dye anion ( $\left.\mathrm{SR}^{-}, \mathrm{EE}^{-}\right)$ and the cationic surfactant. It was found that in the domicellar region of surfactant concentrations, an increase in the concentration of CPB is accompanied by a decrease in the light absorption of the dye. A further increase in the surfactant content (the appearance of micelles) leads to an increase in light absorption with a significant (up to $35 \mathrm{~nm}$ in the case of EE) batochromic shift relative to the light absorption of the aqueous dye solution. This result is consistent with the known data on the influence of cationic surfactants on spectra of the dye. Standard formation enthalpies of ions and associates were calculated by semi-empirical methods (AM1 and PM3) in vacuum and in water. Regardless of the method the calculations show that the interaction " $\mathrm{CP}^{+}+\mathrm{SR}^{-"}$ or " $\mathrm{CP}^{+}+\mathrm{EE}^{-"}$ " is energetically beneficial since the energy gain significantly exceeds the statistical error of the methods. The obtained results made it possible to develop a new method for determination of CMC cationic surfactant in water using SR dye.

\section{Conflict of Interest}

The authors declare that there is no conflict of interest.

\section{References}

[1] S.A. Shapovalov, The association processes of protolytic forms of dyes in solutions. Dissimilar association, Kharkiv National University, Kharkiv, Ukraine, 2014. (in Ukrainian) ISBN 978-966-2445-78-7.

[2] B. Heyne, Self-assembly of organic dyes in supramolecular aggregates, Photochemical \& Photobiological Sciences. 15 (2016) 1103-1114.

[3] S.A. Shapovalov, Processes of self-association of dyes in solutions, Academic Publishing of European Union, OmniScriptum Publishing group, Riga, Latvia, 2018. ISBN 978-613-982294-2.

[4] M.S. Kumar, S. Dash, Effect of salts on micellization and clouding behavior of Pluronic F108 in aqueous solution using Trypan blue dye, Surfaces and Interfaces. 12 (2018) 1-7.

[5] M. Mobarak et al., A superior adsorbent of $\mathrm{CTAB} / \mathrm{H}_{2} \mathrm{O}_{2}$ solution-modified organic carbon rich-clay for hexavalent chromium and methyl orange uptake from solutions. 259 (2018) 384397.

[6] W. Ahmad et al., Enhancing sensitivity by Triton X-100 in extractive spectrophotometric determination of chromium via transforming the formed binary complex ion associate between chromate and rhodamine $6 \mathrm{G}$ into an adduct, Journal of Molecular Liquids. 261 (2018) 225-231. 
[7] M. Kaul, C.M. Barbieri, D.S. Pilch, Fluorescence-based approach for detecting and characterizing antibiotic-induced conformational changes in ribosomal RNA: Comparing aminoglycoside binding to prokaryotic and eukaryotic ribosomal RNA sequences, Journal of the American Chemical Society. 126(11) (2004) 3447-3453.

[8] M. López et al., Importance of hydrophobic interactions in the single-chained cationic surfactant-DNA complexation, Journal of Colloid and Interface Science. 521 (2018) 197-205.

[9] S.A. Shapovalov, Ya.S. Kiseliova, Association of thymolsulfonephthalein and cresolsulfonephthalein anions with cationic cyanines in aqueous solution, Chemistry \& Chemical Technology. 4 (2010) 271-276.

[10] S.A. Shapovalov, Heteroassociates of singly- and doubly-charged anions of alizarin red S with the pinacyanol cation, Russian Chemical Bulletine. 60(1) (2011) 89-98.

[11] V.I. Larin, S.A. Shapovalov, Association between complex ferrumcyanide anions with pinacyanol cation in aqueous solution, Chemistry \& Chemical Technology. 6 (2012) 25-29.

[12] A. Ali, S. Uzair, U. Farooq, Interactions of cationic, anionic and nonionic surfactants with cresol red dye in aqueous solutions: Conductometric, tensiometric, and spectroscopic studies, Tenside, Surfactants, Detergents. 54(4) (2017) 342-352.

[13] S.A. Shapovalov, Cation-anionic association of organic dyes in aqueous solutions: structure and properties of associates, Modern Organic Chemistry Research. 2 (2017) 195-203.

[14] H. Lin et al., Interactions between bromophenol blue and cetyl- trimethylammonium bromide in aqueous solutions and microemulsions, Journal of Dispersion Science and Technology. 38(10) (2017) 1410-1414.

[15] J. Guo et al., Interaction between Coomassie brilliant blue G250 and octylphenol polyoxyethylene ether (10) in aqueous solution, Journal of Dispersion Science and Technology. 39(8) (2018) 1208-1213.

[16] S.A. Shapovalov, Association of quinaldine red cation in an aqueous solution: the interaction with anionic dyes, AASCIT Journal of Nanoscience. 3 (2017) 35-40.

[17] P.C.D.S. Pereira et al., Photophysical properties and interaction studies of Rose Bengal derivatives with biomimetic systems based in micellar aqueous solutions, Journal of Molecular Liquids. 230 (2017) 674-685.

[18] N. Wang et al., Spectroscopic studies of sodium dodecyl sulfate and Sudan red III associations in solutions, AATCC Journal of Research. 4 (2017) 16-21.

[19] S.A. Shapovalov, Y.A. Svishchova, Heteroassociates of pinacyanol cation in aqueous solutions: formation and their interaction with organic multiply charged anions, FrenchUkrainian Journal of Chemistry. 6(1) (2018) 21-30.

[20] Z. Wang et al., Transport of reactive X-3B dye at the interface between cationic surfactantmodified water-quenched blast furnace slag and aqueous solution, Canadian Journal of Chemical Engineering. 96(6) (2018) 1240-1249.

[21] L. Deng et al., Sodium dodecyl sulfate intercalated and acrylamide anchored layered double hydroxides: A multifunctional adsorbent for highly efficient removal of Congo red, Journal of Colloid and Interface Science. 521 (2018) 172-182.

[22] M. Chen, C.T. Jafvert, Application of cross-linked stearic acid nanoparticles with dialysis membranes for methylene blue recovery, Separation and Purification Technology. 204 (2018) $21-29$.

[23] Y. Zhou et al., A novel amphoteric $\beta$-cyclodextrin-based adsorbent for simultaneous removal of cationic/anionic dyes and bisphenol A, Chemical Engineering Journal. 341 (2018) 47-57. 
[24] N.O. Mchedlov-Petrossyan et al., A new application of rhodamine 200B (sulforhodamine B), Dyes and Pigments. 28 (1995) 7-18.

[25] H. Xirong et al., Determination of the critical micelle concentration of cationic surfactants, Journal of Chemical Education. 76(1) (1999) 93.

[26] B. Gohain, R.K. Dutta, Premicellar and micelle formation behavior of dye surfactant ion pairs in aqueous solutions, Journal of Colloid and Interface Science. 323(2) (2008) 395-402.

[27] M. Rashidi-Alavijeh et al., Intermolecular interactions between a dye and cationic surfactants, Colloids and Surfaces. 380 (2011) 119-127.

[28] Hypercube Products http://www.hyper.com/Products/tabid/354/Default.aspx.

[Online]. Available:

[29] S.A. Shapovalov et al., Association of indopolymethine cyanine cations with anions of sulfonephthalein and xanthene dyes in water, Journal of the Brazilian Chemical Society. 16(2) (2005) 232-240.

[30] S.A. Shapovalov, E.A. Samoilov, Regularities of homo- and heteroassociation of the pinacyanol cation in aqueous solution, Russian Chemical Bulletin. 57(7) (2008) 1405-1415.

[31] M.J.S. Dewar, D.M. Storch, Development and use of quantum molecular models. 75. Comparative tests of theoretical procedures for studying chemical reactions, Journal of the American Chemical Society. 107(13) (1985) 3898-3902. 